\title{
Dirac equation for quasi-particles in graphene and quantum field theory of their Coulomb interaction
}

\begin{abstract}
Riazuddin*
National Centre for Physics, Quaid-i-Azam University Campus, Islamabad, 45320 Pakistan

There is evidence for existence of massless Dirac quasi-particles in graphene, which satisfy Dirac equation in $(1+2)$ dimensions near the so called Dirac points which lie at the corners at the graphene's brilluoin zone. We revisit the derivation of Dirac equation in $(1+2)$ dimensions obeyed by quasiparticles in graphene near the Dirac points. It is shown that parity operator in $(1+2)$ dimensions play an interesting role and can be used for defining "conserved" currents resulting from the underlying Lagrangian for Dirac quasi-particles in graphene which is shown to have $U_{A}(1) \times U_{B}(1)$ symmetry. Further the quantum field theory (QFT) of Coulomb interaction of $2 \mathrm{D}$ graphene is developed and applied to vacuum polarization and electron self energy and the renormalization of the effective coupling $g$ of this interaction and Fermi velocity $v_{f}$ which has important implications in the renormalization group analysis of $g$ and $v_{f}$.
\end{abstract}

Recent progress in the experimental realization of a single layer problem of graphene has led to extensive exploration of electronic properties in this system. Experimental and theoretical studies have shown that the nature of quasiparticles in these two-dimensional system are very different from those of the conventional two-dimensional electron gas (2DEG) system realized in the semiconductor heterostructures. Graphene has a honeycomb lattice of carbon atoms. The quasiparticles in graphene have a band structure in which electron and hole bands touch at two points in the Brillouin zone. At these Dirac points the quasiparticles obey the massless Dirac equation. In other words, they behave as massless Dirac fermions leading to a linear dispersion relation $\epsilon_{k}=v k$ (with the characteristic velocity $v \simeq 10^{6} \mathrm{~m} / \mathrm{s}$ ). This difference in the nature of the quasiparticles in graphene from conventional 2DEG has given rise to a host of new and unusual phenomena such as anomalous quantum Hall effects and a $\pi$ Berry phase [1, 2]. These transport experiments have shown results in agreement with the presence of Dirac fermions. The 2D Dirac-like spectrum was confirmed recently by cyclotron resonance measurements and also by angle resolved photoelectron spectroscopy (ARPEC) measurements in monolayer graphene [3]. Recent theoretical work on graphene multilayer has also shown the existence of Dirac electrons

\footnotetext{
* riazuddin@ncp.edu.pk
} 
with a linear energy spectrum in monolayer graphene [4].

In this work, we revisit the derivation of Dirac equation obeyed by quasiparticles in graphene near the Dirac points. Since monolayer graphene is a physical system $(1+2)$ space-time dimensions this has important consequences: Dirac matrices in odd number of space-time dimensions can have two inequivalent representations and their role in obtaining the Dirac equation for quasiparticles is emphasized here. Moreover, it is also shown that the Lagrangian for the system has chiral $U_{A}(1) \otimes U_{B}(1)$ symmetry. Furthermore, we have carried out a QFT calculation of Coulomb interaction effects in graphene. The obtained results are compared with those obtained in [5]. The existence of quasi-particles in graphene, which satisfy massless Dirac equation in $(1+2)$ dimensions near the so called Dirac points, which lie at the corners of graphene's Brilluoin zone. This is indicated by energy dispersion around the Dirac point given by [] $]$

$$
E_{ \pm} \simeq \pm v_{f}|\mathbf{q}|+O\left(|\mathbf{q}|^{2}\right)
$$

which reminds one of relativistic energy-momentum relation for massless or ultra relativistic particles although Fermi velocity is three hundred times smaller than the speed of light. The derivation of such an equation given in literature [6, 7] is in the form

$$
i \sigma . \nabla \psi=E \psi
$$

where $\sigma=\left(\sigma^{1}, \sigma^{2}\right)$ and $\nabla=\left(\frac{\partial}{\partial x^{1}}, \frac{\partial}{\partial x^{2}}\right)$, which has two dimensional form of the usual Dirac equation for two component left-handed or right-hand massless fermions and not exactly in the form of Dirac equation in $(1+2)$ dimensions[see below]. The purpose of this paper is to clarify such points and to discuss the subtleties of odd [in this case three] space-time dimensions and develop a quantum field theory of Coulomb interaction of $2 \mathrm{D}$ graphene.

In graphene for the hexagonal layer the unit cell contains two atoms $\mathrm{A}$ and $\mathrm{B}$, belonging to two sublattices. The lattice vectors are [6]

$$
\mathbf{a}_{1}=\frac{a}{2}(3, \sqrt{3}), \quad \mathbf{a}_{2}=\frac{a}{2}(3,-\sqrt{3})
$$

where $a \simeq 1.42 A^{0}$ is the carbon-carbon distance.

The Dirac points $\mathrm{K}$ and $\mathrm{K}^{\prime}$ have their position vectors in momentum space:

$$
\mathbf{K}=\frac{2 \pi}{3 a}(1, \sqrt{3}), \quad \mathbf{K}^{\prime}=\frac{2 \pi}{3 a}(1,-\sqrt{3})
$$


In the tight binding approach, the Hamiltonian is given by [6]

$$
\mathcal{H}=-t \sum_{<i, j>}\left(a_{i}^{\dagger} b_{j}+\text { h.c. }\right)
$$

where $a_{i}\left(a_{i}^{\dagger}\right)$ are annihilation(creation) operators for an electron in sub-lattice A with an equivalent definition for sub-lattice B. $t(\simeq 2.8 \mathrm{eV})$ is the nearest hopping energy (hopping between different sub-lattices).

Introduce now Fourier transform of field operator:

$$
\begin{aligned}
& a_{i}=\sum_{k} a_{k i} e^{i \vec{k} \cdot \vec{u}_{i}} \\
& b_{j}=\sum_{k} b_{k j} e^{i \vec{k} \cdot \vec{u}_{j}}
\end{aligned}
$$

where.$\vec{u}_{i}$ (or $\vec{u}_{j}$ ) may be taken any of two independent lattice points [2]. $\mathbf{a}_{1}$ and $\mathbf{a}_{2}$ which may taken as in Eq. (3). Then Eq. (5) gives

$$
\mathcal{H}=\sum_{k}\left(a_{k i}^{\dagger} b_{k j}^{\dagger}\right) \mathcal{H}_{D}\left(\begin{array}{l}
a_{k i} \\
b_{k j}
\end{array}\right)
$$

where

$$
\mathcal{H}_{D}=\left(\begin{array}{ll}
0 & \mathcal{H}_{A B} \\
\mathcal{H}_{A B}^{*} & 0
\end{array}\right)
$$

with

$$
\mathcal{H}_{A B}=-t\left[e^{i \mathbf{k} \cdot \mathbf{a}_{1}}+i e^{i \mathbf{k} \cdot \mathbf{a}_{2}}\right]
$$

Let us expand around the Dirac point $K^{\prime}$.

$$
\mathbf{k}=\mathbf{K}^{\prime}+\mathbf{q}
$$

keep terms linear in $|\mathbf{q}|$,

$$
\mathcal{H}_{A B}=\frac{3 a t}{2}\left[i q_{x}+q_{y}\right]
$$

This gives

$$
\begin{aligned}
\mathcal{H}_{D} & =\left(\begin{array}{ll}
0 & i q_{x}+q_{y} \\
-i q_{x}+q_{y} & 0
\end{array}\right) \\
& =v_{f}\left[-q^{1} \sigma^{2}+q^{2} \sigma^{1}\right]
\end{aligned}
$$


where $v_{f}=\frac{3 a t}{2}$ is the Fermi velocity, $\sigma^{1}$ and $\sigma^{2}$ are Pauli matrices, $q^{i}=\left(q^{1}, q^{2}\right)$, in relativistic notation: $q^{\mu}=\left(q^{0}, q^{1}, q^{2}\right)$. This does not give $\sigma \cdot \mathbf{q}$ as implied in Eq. (21). The Eq. (9) can be put in the form

$$
\begin{aligned}
\mathcal{H}_{D} & =v_{f}\left[i \sigma^{3} \sigma^{1} q^{1}+i \sigma^{3} \sigma^{2} q^{2}\right] \\
& =\gamma^{0}\left(\gamma \cdot \mathbf{q} v_{f}\right)
\end{aligned}
$$

where $\gamma^{0}=\sigma^{3}, \gamma^{1}=i \sigma^{1}, \gamma^{2}=i \sigma^{2}$ are Dirac matrices in (1+2) dimensions. The above is the Dirac Hamiltonian for massless fermion in $(1+2)$ dimensions near the Dirac point $K^{\prime}$. The corresponding Dirac equation is

$$
\mathcal{H} \psi=E \psi_{ \pm}, E= \pm v_{f}|\mathbf{q}|
$$

or

$$
i \frac{\partial \psi}{\partial t}=\gamma^{0}\left(\gamma\left(-i \nabla v_{f}\right) \psi\right.
$$

Writing $\partial_{0}=\frac{1}{v_{f}} \frac{\partial}{\partial t}$, we can write the Eq (11) in covariant form

$$
i\left(\gamma^{0} \partial_{0}+\gamma^{1} \partial_{1}+\gamma^{2} \partial_{2}\right) \psi=0
$$

or

$$
i\left(\gamma^{\mu} \partial_{\mu}\right) \psi=0
$$

which is the Dirac equation in $(1+2)$ dimensions for a massless fermion [8].

It is important to remark that if we expand around the Dirac point $\mathrm{K}$, we obtain

$$
\begin{aligned}
\mathcal{H}_{D} & =v_{f}\left[-q^{1} \sigma^{2}-q^{2} \sigma^{1}\right] \\
& =v_{f}\left[i \sigma^{3} \sigma^{1} q^{1} \sigma^{1}-i \sigma^{3} \sigma^{2} q^{2}\right]
\end{aligned}
$$

Now it is known [8] that in 3 space-time dimensions there exists two inequivalent representations for $\gamma$-matrices [this is true for any odd number of space-time dimensions]:

$$
\begin{aligned}
& \gamma^{0}=\sigma^{3}, \gamma^{1}=i \sigma^{1}, \gamma^{2}=i \sigma^{2} \\
& \gamma^{0}=\sigma^{3}, \gamma^{1}=i \sigma^{1}, \gamma^{2}=-i \sigma^{2}
\end{aligned}
$$

We have used the first of these representations for the expansion around the Dirac point $\mathrm{K}^{\prime}$. We take the second representation for the expansion around the Dirac point $\mathrm{K}$, whcih is obtained 
from $\mathrm{K}^{\prime}$ by the parity operation, defined by the matrix [8]

$$
\Lambda=\left(\begin{array}{lll}
1 & 0 & 0 \\
0 & 1 & 0 \\
0 & 0 & -1
\end{array}\right)
$$

so that $\operatorname{det} \Lambda=-1$. Thus we see that under the parity operation

$$
q^{1} \longleftrightarrow q^{1}, q^{2} \longleftrightarrow-q^{2} \text { and } \mathcal{H}_{K} \longleftrightarrow \mathcal{H}_{K^{\prime}}
$$

Taking the two representations mentioned above into account we can write the parity conserving Lagrangian [8] as (it is instructive to put mass term which can be put equal to zero when necessary)

$$
\mathcal{L}=\bar{\psi}_{+}\left(i \partial-m v_{f}^{2}\right) \psi_{+}+\bar{\psi}_{-}\left(i \widetilde{\partial}-m v_{f}^{2}\right) \psi_{-}
$$

where

$$
\begin{aligned}
& \partial=\gamma^{0} \partial_{0}+\gamma^{1} \partial_{1}+\gamma^{2} \partial_{2} \\
& \widetilde{\partial}=\gamma^{0} \partial_{0}+\gamma^{1} \partial_{1}-\gamma^{2} \partial_{2}
\end{aligned}
$$

Parity operation takes the solutions in one representation to the other:

$$
\begin{aligned}
& \psi_{+}^{p}\left(x^{p}\right)=-\eta_{p} \psi_{-}(x) \\
& \psi_{-}^{p}\left(x^{p}\right)=-\eta_{p} \psi_{+}(x)
\end{aligned}
$$

where $x^{p}=\left(x^{0}, x^{1},-x^{2}\right)$. It is convenient to transform to new fields [8]

$$
\begin{aligned}
& \psi_{A}=\psi_{+} \\
& \psi_{B}=i \gamma^{2} \psi_{-}
\end{aligned}
$$

The Lagrangian (17) can then be written as [8]

$$
\mathcal{L}=\bar{\psi}_{A}\left(i \gamma^{\mu} \partial_{\mu}-m v_{f}^{2}\right) \psi_{A}+\bar{\psi}_{B}\left(i \gamma^{\mu} \partial_{\mu}+m v_{f}^{2}\right) \psi_{B}
$$

where under partiy operation now

$$
\begin{aligned}
\psi_{A}^{p}\left(x^{p}\right) & =-i \eta_{p} \gamma^{2} \psi_{B}(x) \\
& =\eta_{p} \sigma^{2} \psi_{B}(x) \\
\psi_{B}^{p}\left(x^{p}\right) & =-i \eta_{p} \gamma^{2} \psi_{A}(x) \\
& =\eta_{p} \sigma^{2} \psi_{A}(x)
\end{aligned}
$$


It may be noted that the Lagrangian (20) is invariant under two independent transformations

$$
\psi_{A} \rightarrow e^{i \alpha_{A}} \psi_{A}, \psi_{B} \rightarrow e^{i \alpha_{B}} \psi_{B}
$$

where $\alpha_{A}$ and $\alpha_{B}$ are real, and has thus $U_{A}(1) \otimes U_{B}(1)$ symmetry. The corresponding conserved currents are

$$
\begin{aligned}
J_{A}^{\mu} & =\bar{\psi}_{A} \gamma^{\mu} \psi_{A} \\
J_{B}^{\mu} & =\bar{\psi}_{B} \gamma^{\mu} \psi_{B}
\end{aligned}
$$

When can form even (odd) combinations corresponding to "vector" ("axial vector") under parity

$$
J_{ \pm}=\left(\bar{\psi}_{A} \gamma^{\mu} \psi_{A} \pm \bar{\psi}_{B} \gamma^{\mu} \psi_{B}\right)
$$

We can develop quantum field theory for Coulomb interaction of 2D graphene in analogy with QED (for another approach see [7, 9]). The "free" Hamiltonian as implied by Eq. (12) is

$$
\begin{aligned}
\mathcal{H}_{o} & =v_{f} \int d^{2} r \psi^{\dagger}(\mathbf{r}) \gamma^{o} \gamma \cdot(-i \nabla) \psi(\mathbf{r}) \\
& =v_{f} \int d^{2} r \bar{\psi}(\mathbf{r}) \gamma \cdot(-i \nabla) \psi(\mathbf{r})
\end{aligned}
$$

The instantaneous Coulomb interaction in 2D graphene is

$$
\mathcal{H}_{I}=\frac{e^{2}}{2} \int d^{2} x_{1} d^{2} x_{2} \frac{n\left(t, \mathbf{x}_{1}\right) n\left(t, \mathbf{x}_{2}\right)}{4 \pi\left|\mathbf{x}_{1}-\mathbf{x}_{2}\right|}
$$

where $n\left(t, \mathbf{x}_{1}\right)=\psi^{\dagger}\left(t, \mathbf{x}_{1}\right) \psi\left(t, \mathbf{x}_{1}\right)=\bar{\psi}\left(t, \mathbf{x}_{1}\right) \gamma^{o} \psi\left(t, \mathbf{x}_{1}\right)$. This gives the scattering matrix element between four fermions

$$
S_{f i}^{\text {coulomb }}=-i e^{2} \frac{1}{2} \int d t \int d^{2} x_{1} \int d^{2} x_{2} \frac{<f\left|n\left(t, \mathbf{x}_{1}\right) n\left(t, \mathbf{x}_{2}\right)\right| i>}{4 \pi\left|\mathbf{x}_{1}-\mathbf{x}_{2}\right|}
$$

The integral can be written as $\int d^{D} x_{1} d^{D} x_{2} \delta\left(t_{2}-t_{1}\right)$ where $D=3$ in $(1+2)$ dimensions. Expanding $\psi^{\prime}$ s into creation and annihilation operators, one finally obtains [10]

$$
i T_{f i}=\frac{1}{4 \pi v_{f}}\left[\bar{u}\left(p_{2}^{\prime}\right)\left(-i e \gamma^{\mu}\right) u\left(p_{2}\right) \frac{i \eta_{\mu} \eta_{\nu}}{2\left[-q^{2}+(q \cdot \eta)^{2}\right]^{\frac{1}{2}}} \bar{u}\left(p_{1}^{\prime}\right)\left(-i e \gamma^{\mu}\right) u\left(p_{1}\right)-\text { crossed term }\right]
$$

where $\frac{1}{2|\mathbf{q}|},|\mathbf{q}|=\left[-q^{2}+(q \cdot \eta)^{2}\right]^{1 / 2}$ is Fourier transform of $\frac{1}{4 \pi\left|\mathbf{x}_{1}-\mathbf{x}_{2}\right|}$ in two space dimensions, $\eta^{\mu}=$ $(1,0,0)$, so that $\eta_{\mu} \gamma^{\mu}=\gamma^{o}$ and longitudinal photon momentum may be taken as $l^{\mu} \equiv 2\left(q^{\mu}-\eta . q \eta^{\mu}\right)$, $\mu=0,1,2$; so that $\left(-l^{2}\right)^{1 / 2}=2|\mathbf{q}|$.

Thus we may write Feynman rules for 2D coulomb interaction in analogy with QED as follows: 
(i) Vertex factor: $-i e \gamma^{\mu}$,

(ii) Internal lines

Photon ("longitudinal") line: $\frac{i \eta_{\mu} \eta_{\nu}}{2\left[-q^{2}+(q \cdot \eta)^{2}\right]^{\frac{1}{2}}}$

(iii) Spin $\frac{1}{2}$ massless fermion: $\frac{i}{\not p}$

$\not p=\gamma^{\mu} p_{\mu} \mu=0,1,2$, this follows from the Lagrangian given in Eq. (20).

As an application of these rules, we calculate vacuum polarization $\operatorname{II}\left(q^{2}\right)=-\eta_{\mu} \eta_{\nu} \operatorname{II}^{\mu \nu}(q)$, which arises from photon self energy due to fermion loop. It is given by

$$
-i \mathrm{II}^{\mu \nu}(q)=2(-1) \frac{1}{v_{f}} \int \frac{d^{D} l}{(2 \pi)^{D}} \operatorname{Tr}\left[\left(-i e \gamma^{\mu}\right) \frac{i}{\not+i \epsilon}\left(-i e \gamma^{\nu}\right) \frac{i}{\not p+2(\not q-q \cdot \eta) \not \eta+i \epsilon}\right]
$$

where the factor of 2 arises due to the two terms in Lagrangian (??). The integration will be done by using Feynman parametrization and dimensional regularization. Taking the trace in Eq. (29), using the Feynman parametrization and making the shift $l \rightarrow l-2 q x+2(q-\eta) \eta x$, we obtain

$$
-i \mathrm{II}^{\mu \nu}(q)=-2 i \frac{D+1}{2} \frac{e^{2}}{v_{f}} \int_{0}^{1} d x \int \frac{d^{D} x}{(2 \pi) D}\left[\left(2 l^{\mu} l^{\nu}-g^{\mu \nu} l^{2}\right)+g^{\mu \nu} L+x(x-1) A^{\mu \nu}\right] \frac{1}{\left(l^{2}-L\right)^{2}}
$$

where $\left(\frac{D+1}{2}\right)$ arises from the trace for odd space time dimensions, $L=4\left[(q \cdot \eta)^{2}-q^{2}\right]$ and

$$
A^{\mu \nu}=-8 g^{\mu \nu}\left((q \cdot \eta)^{2}-q^{2}\right)-8 q^{\mu} q^{\nu}+8 q \cdot \eta\left(q^{\mu} \eta^{\nu}+\eta^{\nu} q^{\mu}-(q \cdot \eta) \eta^{\mu} \eta^{\nu}\right)
$$

The contribution from singular terms $\left(2 l^{\mu} l^{\nu}-g^{\mu \nu} l^{2}\right)$ combines to give $-g^{\mu \nu}(1-D / 2) \Gamma(1-D / 2) L=$ $-g^{\mu \nu} \Gamma(2-D / 2) L$, i.e. finite answer, which however cancels with the contribution from the second term in parenthesis in Eq. (30). The net result is

$$
\mathrm{II}^{\mu \nu}\left(q^{2}\right)=\frac{2 e^{2}}{v_{f}} \frac{1}{(2 \pi)^{\frac{D}{2}}}\left(\frac{D+1}{2}\right) \Gamma\left(2-\frac{D}{2}\right) \int_{0}^{1} d x \sqrt{x(1-x)} \frac{A_{\mu \nu}}{2 \Delta}
$$

where $\Delta=\left[(q \cdot \eta)^{2}-q^{2}\right]^{\frac{1}{2}}=|\mathbf{q}|$.

Using $D=3$, we finally get

$$
\begin{aligned}
\mathrm{II}\left(q^{2}\right) & =-\eta_{\mu} \eta_{\nu} \mathrm{II}^{\mu \nu}=\frac{e^{2}}{4 v_{f}}(\Delta) \\
& =\frac{e^{2}}{4 \pi v_{f}}|\mathbf{q}| \pi \\
& =g \pi|\mathbf{q}|
\end{aligned}
$$

in agreement with the known result [6,7], where $g=\frac{e^{2}}{4 \pi v_{f}}$ is the dimensionless [in units $\hbar=1$ ] effective coupling constant.

We now discuss how the vacuum polarization renormalizes the interaction coupling constant. For this purpose we consider the response of charged fermion to an externally applied field [Coulomb 
potential in two space dimensions in momentum space is $A_{o}=-\frac{e}{2|\mathbf{q}|}=\eta_{\mu} A^{\nu}$, where $A^{\nu}=\left(-\frac{e}{2|\mathbf{q}|}\right.$, 0)], namely

$$
\bar{u}\left(-i e \gamma^{\nu}\right) u i A_{\nu}=\bar{u}\left(-i \gamma^{0}\right) u\left(-e^{2} / 2|\mathbf{q}|\right)
$$

which is modified to

$$
\begin{aligned}
& \bar{u}\left(-i e \gamma^{0}\right) u A_{0}\left[\frac{1}{1-\eta_{\lambda} \eta \rho \mathrm{II} \mathrm{I}^{\lambda \rho} /(2 \Delta)}\right] A_{\nu} \\
= & \bar{u}\left(-i \gamma^{0}\right) u\left(-e^{2} / 2|\mathbf{q}|\right) 1 /\left[1+\mathrm{II}\left(q^{2}\right) / 2 \Delta\right] \\
= & \bar{u}\left(-i \gamma^{0}\right) u\left[-g \frac{4 \pi v_{f}}{2|\mathbf{q}|} \frac{1}{1+\frac{g \pi}{2}}\right]
\end{aligned}
$$

where we have used Eq. (33). Thus the renormalized $g$, often written as $g_{s c}$ [11] is

$$
g_{s c}=\frac{g}{1+\frac{g \pi}{2}}
$$

The implications of this result are discussed in [6,7]. It is instructive to calculate fermion self energy which is given by

$$
-i \Sigma(p)=\frac{1}{v_{f}} \int \frac{d^{3} l}{(2 \pi)^{3}}\left(-i e \gamma^{\mu}\right) \frac{i}{\not}\left(-i e \gamma^{\nu}\right) \frac{i \eta_{\mu} \eta_{\nu}}{2|\mathbf{l}-\mathbf{p}|}
$$

After making the Wick rotation $l^{0}=i l_{E}^{0}$ ( $E$ for Euclidean matric) and carrying out the $l_{E}^{0}$ integration (which occurs only in fermion propagator), which gives $\pi \frac{1}{\mid 1}$, the rest of integration is in $D=2$ dimension. Using Feynman parametrization

$$
\frac{1}{a^{1 / 2} b^{1 / 2}}=\frac{1}{\pi} \int_{0}^{1} x^{-1 / 2}(1-x)^{-1 / 2} \frac{1}{a x+b(1-x)},
$$

and the dimensional regularization one obtains

$$
\begin{aligned}
-i \Sigma(p) & =-\frac{i e^{2}}{16 \pi v_{f}} \int_{0}^{1} x^{-1 / 2}(1-x)^{-1 / 2} \int \frac{d^{D} l}{(2 \pi)^{D}} \frac{-\not \eta \not p \not h x}{\left(\mathbf{l}^{2}-L\right)} \\
& =-\frac{i e^{2}}{4 \pi v_{f}} \int_{0}^{1} x^{1 / 2}(1-x)^{-1 / 2} \frac{1}{(4 \pi)^{D}} \frac{\Gamma(1-D / 2)}{\Gamma(1)}\left(\frac{1}{L}\right)^{1-D / 2} \\
& =-\frac{i e^{2}}{32 \pi v_{f}}\left(-\not \gamma \gamma \cdot \mathbf{p} \not \text { h) } \ln \frac{\Lambda^{2}}{|\mathbf{p}|^{2}}\right.
\end{aligned}
$$

where we have used

$$
\frac{1}{(4 \pi)^{(D / 2-1)}} \frac{\Gamma(1-D / 2)}{\Gamma(1)}\left(\frac{1}{L}\right)^{1-D / 2}=\left[\frac{2}{\varepsilon}-(\ln L+\gamma-\ln 4 \pi)\right]
$$

with $L=|\mathbf{p}|^{2} x(1-x), \frac{2}{\varepsilon}$ signifies the ultraviolet log divergence: $\ln \Lambda^{2}$. 
Noting that

$$
\begin{aligned}
-\not \eta \gamma \cdot p \not h & =\gamma \cdot \mathbf{p}=-\not p+\gamma^{0} p^{0} \\
\Sigma(p) & =\frac{g}{4}\left[-\not p+\gamma^{0} p^{0}\right] \ln \frac{\Lambda}{|\mathbf{p}|}
\end{aligned}
$$

where $p^{0}=v_{f}|\mathbf{p}|$ is the energy. The usual interpretation of this result is that coefficient of second term in parenthesis gives the radiative correction to energy

$$
E=v_{f}|\mathbf{p}| Z_{2}^{-1}
$$

where

$$
Z_{2}^{-1}=1+\frac{g}{4} \ln \frac{\Lambda}{|\mathbf{p}|}
$$

The coefficient of $\not p$ gives the renormalization of the electric charge, $e \rightarrow Z_{2} e$, which however is canceled by the corresponding contribution from the vertex part by the use of Ward Identity. The vacuum polarization correction is finite and renormalize $g$ to $g_{s c}$ as given in Eq. (36). The result (41) is interpreted as renormalization of the Fermi velocity $v_{f}$ [7, 9, 11]. Thus [putting $|\mathbf{p}|=q$ ]

$$
v_{f}(q)=v_{f 0} Z_{2}^{-1}=v_{f 0}+v_{f 0} \frac{g}{4} \ln \frac{\Lambda}{q} .
$$

We remove the cut-off and unrenormalized $v_{f 0}$ by subtraction at $q=q_{0}$ :

$$
v_{f}(q)-v_{f}\left(q_{0}\right)=\frac{g}{4} v_{f 0} \ln \frac{q_{0}}{q}=\frac{g}{4} v_{f}\left(q_{0}\right) \ln \frac{q_{0}}{q}+O\left(g^{2}\right)
$$

Since

$$
g=\frac{e^{2}}{4 \pi v_{f}}
$$

this gives [11]

$$
g(q)=g_{0}\left[1+\frac{g_{0}}{4} \ln \frac{q_{0}}{q}\right]^{-1}=\left[g_{0}^{-1}+\frac{1}{4} \ln \frac{q_{0}}{q}\right]^{-1}
$$

where $g_{0}=g\left(q_{0}\right)$. One may take $q_{0}$ as the inverse of the lattice constant [for our case $q_{0}=4 n m^{-1}$ ]. An application of this result has been discussed in [5].

In summary we have clarified the derivation of Dirac equation for quasi-particles in graphene in $(1+2)$ dimensions near the Dirac points $K^{\prime}$ and $K$. The role of two inequivalent representations of Dirac matrices in $(1+2)$ dimensions [a property of odd number of space-time dimensions] visa vis parity operation is emphasized. It is shown that the underlying Lagrangian for quasi-particles in 
graphene has chiral $U_{L}(1) \otimes U_{R}(1)$ symmetry. Further Feynman rules for QFT of Coulomb interaction of 2D graphite have been given and applied to the vacuum polarization and renormalization of effective Coulomb interaction constant and electron self energy which has important implication in the renormalization group analysis of $g$ and $v_{f}[11]$.

The author would like to thank Ansar Fayyazuddin for introducing him to this subject by giving him the reference for the review article [6] . He would also like to express his deep appreciation to Kashif Sabeeh for reading this manuscript and making some useful suggestions. Author would also like to acknowledge the hospitality of King Fahad University of Patroleum and Minerals, Dhahran where a part of the work was completed.

[1] K. S. Novoselov, A. K. Geim, S. V. Morozov, D. Jiang, M. I. Katsnelson, I. V. Grigorieva, S. V. Dubonos and A. A. Firsov, Nature 438, 197 (2005); Y. Zhang, Y. W. Tan, H. L. Stormer and P. Kim, Nature $438,201(2005)$.

[2] Y. Zheng and T. Ando, Phys. Rev. B 65, 245420 (2002); V. P. Gusynin and S. G. Sharapov, Phys. Rev. Lett. 95, 146801 (2005); N.M. R. Perez, F. Guinea, and A. H. Castro Neto, PhysA. Rev. B 73, 125411, (2006); M. I. Kasnelson, K. S. Novoselov and A. K. Geim, Nat. Phys. 2, 620 (2006); K. S. Novoselov, E. McCann, S. V. Morozov, V. I. Fal'ko, M. I. Katsnelson, U. Zeitler, D. Jiang, F. Schendin and A. K. Geim, Nat. Phys. 2, 177 (2006).

[3] R. S. Deacon, K-C. Chuang, R. J. Nicholas, K. S. Novoselov and A. K. Geim, arXiv: 0704.0410v3; S. Y. Zhou, G.-H. Gweon, J. Graf, A. V. Fedorov, C. D. Spataru, R. D. Diehl, Y. Kopelevich, D. H. Lee, S. G. Louie, and A. Lanzara, Nat. Phys. 2, 595 (2006).

[4] B. Partoens and F. M. Peeters, Phys. Rev. B 75, 193402 (2007).

[5] C. L. Kane and E. J. Mele, Phys. Rev. Lett. 93, 197402 (2004).

[6] A.H.Castro Neto, G.Guinea, N.M.R. Peres, K.S.Novoselov, and A.K. Gein, The electronic properties of graphene, arXiv:0709.1163v1 1 [cond.mat.other], 7 Sep., 2007.

[7] F. Guinea, M. Pilar Lopez-Sancho, and Maria A. H. Vorzmediano, Intractions and disorder in 2D graphite sheets, arXiv: 0511558v1[cond-mat.str.ef], 22 Nov, 2005

[8] See for a review, lecture notes of a short course given on "Origin of Mass" by Adnan Bashir at National Centre for Physics, Quaid-I-Azam University, Islamabad, in Dec. 2005.

[9] J.Gonzalez, F. Guinea and M. A. H. Vormediano, Phys. Rev. B59, R 2474, 1999.

[10] See for example, J. J. Sakurai, Advanced Quantum Mechanics, Addison-Wesley, 1973, p 252,253.

[11] R. R. Biswas, S. Sachdev and D. T. Son, arXiv: 0706-3907. 